\title{
Mutagenesis of charged residues in a conserved sequence in the 2-kinase domain of 6-phosphofructo-2-kinase/fructose-2,6-bisphosphatase
}

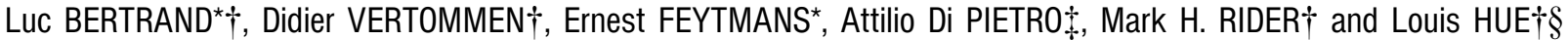 \\ *Facultés Universitaires Notre-Dame de la Paix, Department of Biology, rue de Bruxelles, 61, B-5000 Namur, Belgium, †Hormone and Metabolic Research Unit, \\ University of Louvain Medical School, and International Institute of Cellular and Molecular Pathology, Avenue Hippocrate, 75, B-1200 Brussels, Belgium, \\ and \$Institut de Biologie et de Chimie des Protéines, Centre National de la Recherche Scientifique, Passage du Vercors, F-69367 Lyon cedex 07, France
}

\begin{abstract}
Arg-136, Glu-137, Arg-138 and Arg-139 are conserved in all sequences of the 2-kinase domain of 6-phosphofructo-2-kinase/ fructose-2,6-bisphosphatase. Their role was studied by sitedirected mutagenesis. All the mutations had little, if any, effect on fructose-2,6-bisphosphatase activity. Mutations of Arg-136 and Glu-137 into Ala caused only minor modifications of phosphofructo-2-kinase activity. In contrast, mutation of Arg138 into Ala increased 280-fold the $K_{\mathrm{m}}$ for fructose 6-phosphate of phosphofructo-2-kinase. Mutation of Arg-139 into Ala resulted in decreases in phosphofructo-2-kinase $V_{\max } / K_{\mathrm{m}}$ for MgATP and fructose 6-phosphate 600-fold and 5000-fold re-
\end{abstract}

spectively. Mutation of Arg-139 into Lys and Gln increased the $K_{\mathrm{m}}$ of phosphofructo-2-kinase for MgATP (20-fold and 25-fold respectively) and for fructose 6-phosphate (8-fold and 13-fold), and the $\mathrm{IC}_{50}$ for MgADP (30-fold and 50-fold) and for magnesium citrate (7-fold and 25-fold). However, these two mutations did not affect nucleotide binding, as measured by quenching of intrinsic fluorescence. The changes in kinetic properties induced by mutations could not be attributed to structural changes. It is proposed that Arg-138 is involved in fructose 6-phosphate binding and that Arg-139 is probably involved in the stabilization of the transition state and so participates in catalysis.

\section{INTRODUCTION}

The bifunctional enzyme 6-phosphofructo-2-kinase/fructose2,6-bisphosphatase (PFK-2/FBPase-2) catalyses the synthesis and breakdown of fructose 2,6-bisphosphate (Fru-2,6- $\mathrm{P}_{2}$ ), a regulator of glycolysis (reviewed recently in [1]). Isoenzymes of PFK-2/FBPase-2 have been characterized in eukaryotes, including yeast and mammalian cells. They have been termed the L (liver), M (muscle), H (heart), T (testis), B (brain) and pfk26 and fbp26 (yeast) isoenzymes, and several amino acid sequences are available [2-12]. The PFK-2/FBPase-2 isoenzymes differ in their kinetic properties, molecular masses and responses to phosphorylation by protein kinases. They are homodimers and each subunit contains two separate catalytic domains: an Nterminal 2-kinase domain and a C-terminal 2-phosphatase domain. The FBPase-2 domain has been shown to be homologous with the phosphoglycerate mutases [13] and its threedimensional structure has recently been solved [14]. The FBPase2 reaction involves a Ping Pong mechanism in which the phosphoacceptor is a histidine residue (His-258; in this paper we use the numbering of amino acids of the L isoenzyme because the sequences of the $\mathrm{L}$ and $\mathrm{M}$ isoenzymes are identical except for the first 32 residues in the $\mathrm{L}$ isoenzyme, which are replaced by a unique nonapeptide in the $\mathrm{M}$ isoenzyme) $[15,16]$.

The PFK-2 reaction follows a ternary complex mechanism with direct in-line transfer of the $\gamma$-phosphate of MgATP to the C-2 hydroxy group of fructose 6-phosphate (Fru-6-P) [17-19]. Several residues involved in substrate binding in the 2-kinase domain have been identified by site-directed mutagenesis [1]. For example, Arg-104, Arg-195 and Arg-225 participate in Fru-6-P binding [20,21], whereas Lys-54 [22], Arg-230 and Arg-238 [1] bind MgATP.

Alignment of the PFK-2/FBPase-2 isoenzyme sequences has revealed certain well-conserved segments in the PFK-2 domain. Figure 1 shows such a sequence segment containing five charged residues, Asp-130, Arg-136, Glu-137, Arg-138 and Arg-139. These residues might be implicated in catalysis or substrate binding in PFK-2, because negatively charged residues are putative base catalysts for the activation of the hydroxy group of Fru-6-P, whereas positively charged residues could bind the phosphate groups of the two substrates or stabilize the transition state. Previous work from our laboratory suggested that Asp-130 is located in a Walker B motif [23] and participates in $\mathrm{Mg}^{2+}$ binding of the MgATP complex [24].

Here we report the effect of mutation of the four other charged residues $\left(\mathrm{R}^{136} \mathrm{ERR}{ }^{139}\right)$ of this conserved sequence on the kinetic

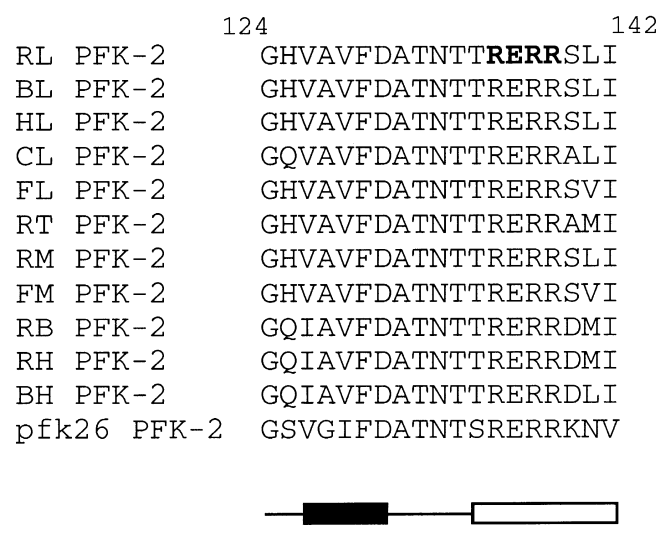

Figure 1 Alignment of a conserved segment of the PFK-2 sequences

Sequences from rat liver (RL), bovine liver (BL), human liver $(\mathrm{HL})$, chicken liver $(\mathrm{CL})$, frog liver $(\mathrm{FL})$, rat testis $(\mathrm{RT})$, rat muscle $(\mathrm{RM})$, frog muscle $(\mathrm{FM})$, rat brain $(\mathrm{RB})$, rat heart $(\mathrm{RH})$, bovine heart (BH) and yeast (pfk26) are aligned. Residues in bold correspond to residues mutated in this work. A predicted $\alpha$-helix (open rectangle) and $\beta$-strand (filled rectangle) are also shown. The numbering of residues corresponds to the rat $L$ isoenzyme. 
properties of PFK-2/FBPase-2. Each residue was first replaced by Ala to obtain four mutants: R136A, E137A, R138A and R139A. Alanine was chosen because (i) it eliminates the side chain beyond the $\beta$ carbon without altering the main-chain conformation, (ii) it does not impose electrostatic or steric interaction, (iii) it is the most abundant amino acid, (iv) it is found frequently both in buried or exposed positions, and (v) it is present in various secondary structures $[25,26]$. Arg-139 was also mutated into similar residues, Lys and Gln. The study was performed on the rat $\mathrm{M}$ and $\mathrm{L}$ isoenzymes.

\section{MATERIALS AND METHODS}

\section{Materials}

All materials and reagents were from sources described [22].

\section{Oligonucleotide-directed mutagenesis}

Four mutants, R136A, E137A, R138A and R139A, were made by annealing the single-stranded DNA template [27] from pBluescript (KS) $\mathrm{II}^{+} / \mathrm{PFK} 2 \mathrm{M}$ to the appropriate mutagenic oligodeoxynucleotides shown in Table 1 . All mutants were obtained as described [28] and identified by sequencing [29]. The selected mutants were introduced into the wild-type expression vector pET/PFK2M-sf [30]. They were called [R136A]M, [E137A]M, [R138A]M and [R139A]M. Mutation of Arg-139 into Ala was also introduced into the expression vector pET/PFK2L-(H) [where $(\mathrm{H})_{6}$ is a C-terminal polyhistidine tail] [22] to give [R139A]L-(H) ${ }_{6}$. The mutations of Arg-139 to Lys and Gln were made by using the single-stranded DNA template from pBlueScript (KS)II ${ }^{+} / \mathrm{PFK} 2 \mathrm{~L}-(\mathrm{H})_{6}$, introduced into the wild-type expression vector $\mathrm{pET} / \mathrm{PFK} 2 \mathrm{~L}-(\mathrm{H})_{6}$ to give $[\mathrm{R} 139 \mathrm{~K}] \mathrm{L}-(\mathrm{H})_{6}$ and [R139Q]L-(H) $)_{6}$.

\section{Expression and purification of recombinant wild-type and mutant PFK-2/FBPase-2 forms}

Wild-type and mutant forms of PFK-2/FBPase-2 were expressed in Escherichia coli BL21(DE3)pLysE. Cultures (2 litres) were grown and induced as described [30]. Bacteria were harvested and lysed for fractionation with 5-20\% (w/v) poly(ethylene glycol) (PEG) 8000 [30].

The [R136A]M, [E137A]M, [R138A]M, [R139A]M and wildtype $\mathrm{M}$ were purified by fractionation with PEG, and chromatography on DEAE-Trisacryl, Blue Sepharose and phosphocellulose [24].

The $[\mathrm{R} 139 \mathrm{~A}, \mathrm{~K}, \mathrm{Q}] \mathrm{L}-(\mathrm{H})_{6}$ mutants were purified by PEG fractionation, chromatography on Q-Sepharose and specific elution from $\mathrm{Ni}^{2+}$-nitrilotriacetic acid-agarose [22]. The active fractions were concentrated by ultrafiltration, dialysed against 200 vol. of
$20 \mathrm{mM}$ Hepes (pH 7.5)/50 mM $\mathrm{KCl} / 5 \mathrm{mM} \quad \mathrm{MgCl}_{2} / 5 \mathrm{mM}$ EDTA/1 mM EGTA/1 mM potassium phosphate $/ 15 \mathrm{mM} 2$ mercaptoethanol $/ 20 \%(\mathrm{v} / \mathrm{v})$ glycerol for $24 \mathrm{~h}$ and stored at $-80{ }^{\circ} \mathrm{C}$.

\section{Measurement of ligand binding by fluorescence}

Binding of ATP and ADP and their $3^{\prime}-N$-methylanthraniloyl (Mant) derivatives was studied by fluorescence quenching [22]. The nucleotide analogues were used because the addition of ATP or ADP to PFK-2/FBPase-2 caused only a $5 \%$ quenching of the intrinsic fluorescence of the enzyme. In the Mant derivatives, a hydrophobic fluorescent moiety is attached to the ribose ring, leaving the purine ring and phosphate groups free for binding. These analogues caused a $50 \%$ quenching of fluorescence, thus allowing a more accurate estimation of binding constants [22,31-34]. Spectra were recorded at $25^{\circ} \mathrm{C}$ in an SLM 8000C spectrofluorimeter (SLM Aminco) [22]. Curve fitting of concentration-dependent changes in fluorescence was performed with Grafit (Erithacus Software) [31,32].

\section{Dissociation and inactivation by guanidinium chloride (GdmCl)}

Structural changes of the wild-type and mutant isoenzymes were assessed by measuring the intrinsic fluorescence of the preparations $(10 \mu \mathrm{g}$ of protein), which were incubated with various concentrations of $\mathrm{GdmCl}$ in a buffer containing $50 \mathrm{mM}$ Hepes, $\mathrm{pH} 7.5,100 \mathrm{mM} \mathrm{KCl}, 1 \mathrm{mM}$ dithiothreitol, $0.2 \mathrm{mM}$ EDTA and $2 \mathrm{mM}$ potassium phosphate. Fluorescence was recorded in a Perkin-Elmer LS-5 luminescence spectrometer from 300 to $400 \mathrm{~nm}$ with an excitation wavelength of $295 \mathrm{~nm}$ [24].

Inactivation of PFK-2 and FBPase-2 by $\mathrm{GdmCl}$ was also measured [21]. The enzyme preparations were incubated in a final volume of $20 \mu \mathrm{l}$ in buffer containing $50 \mathrm{mM}$ Hepes, $\mathrm{pH} 7.5$, $100 \mathrm{mM} \mathrm{KCl}, 0.2 \mathrm{mM}$ EDTA, $2 \mathrm{mM}$ potassium phosphate, $1 \mathrm{mM}$ dithiothreitol and various concentrations of $\mathrm{GdmCl}$. The time course of inactivation by $\mathrm{GdmCl}$ was tested. Maximal inactivation was observed within a few minutes and remained constant for at least $30 \mathrm{~min}$. A similar time course was observed for the changes in fluorescence (results not shown). Therefore the residual activity was assayed as described in the figure legend, after incubation for $15 \mathrm{~min}$ at room temperature.

\section{Other methods}

CD spectra were recorded in a Jobin-Yvon CD6 dichrograph [22] and analysed with the Jobin-Yvon software (version 1.1, 1989).

Protein was measured by the method of Bradford [35] with $\gamma$ globulin as a standard (kinetic measurements) or, after precipitation with trichloroacetic acid in the presence of deoxy-

Table 1 Oligodeoxynucleotides used for site-directed mutagenesis

The underlined codons in the wild-type enzyme represent the sites of mutagenesis. The underlined codon in each mutant codes for the mutated amino acid.

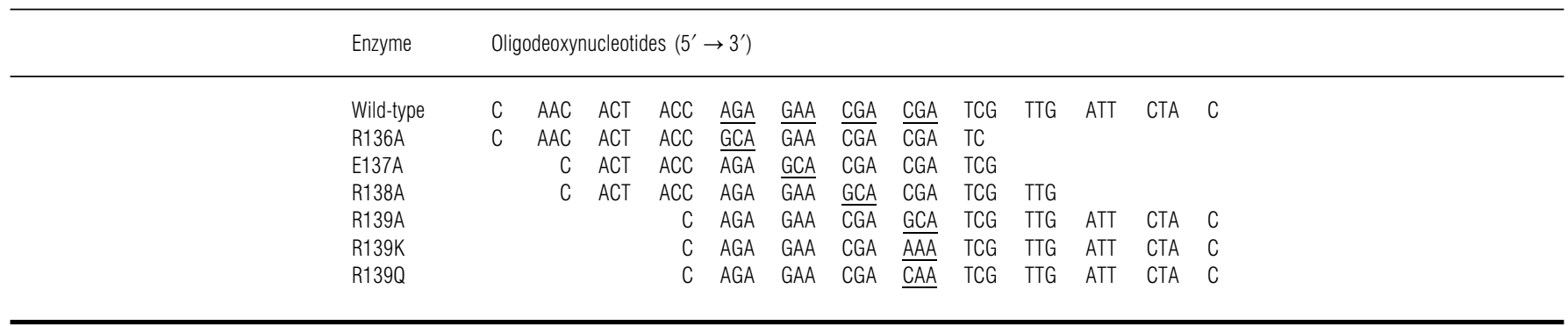


cholate [36], by the method of Lowry et al. [37] with BSA as a standard (fluorescence and CD measurements). PFK-2 and FBPase-2 were assayed as described in the legends to the figures and tables. SDS/PAGE analysis in $10 \%(\mathrm{v} / \mathrm{v})$ polyacrylamide was performed as described [38]. The kinetic data from each single experiment were analysed individually by non-linear leastsquares fitting to a hyperbola describing the Michaelis-Menten equation; this was done with the computer program Ultrafit (Biosoft). The kinetic parameters thus obtained were then averaged to evaluate the reproducibility of the measurements; the means \pm S.E.M. are shown. An unpaired $t$ test was used to evaluate the significance of the differences.

\section{RESULTS}

\section{Purification}

The same protocol, involving PEG fractionation, ion-exchange chromatography on DEAE-Trisacryl, affinity chromatography on Blue Sepharose and chromatography on phosphocellulose, was used to purify the recombinant wild-type $M$ isoenzyme and the four mutants [R136A]M, [E137A]M, [R138]M and [R139A]M. The same chromatographic behaviour was observed with each preparation except for the [R139A]M mutant, which could not be purified. This was because of the low level of expression of this recombinant protein and because of the lack of purification after affinity chromatography on Blue Sepharose. Therefore we resorted to an alternative strategy based on the addition of a C-terminal polyhistidine tail, $(\mathrm{H})_{6}$, and we used the $\mathrm{L}$ isoenzyme, already available as a polyhistidine-tagged form in our laboratory [22]. The [R139A]L-(H) ${ }_{6}$ mutant and two additional mutants, $[\mathrm{R} 139 \mathrm{~K}] \mathrm{L}-(\mathrm{H})_{6}$ and $[\mathrm{R} 139 \mathrm{Q}] \mathrm{L}-(\mathrm{H})_{6}$, were generated. The chromatographic behaviour was the same for the wild-type- $(\mathrm{H})_{6}$ and the mutants. They were eluted from the Q-Sepharose as a single peak at approx. $250 \mathrm{mM} \mathrm{KCl}$, and from the $\mathrm{Ni}^{2+}$-nitrilotriacetic acid-agarose at approx. $160 \mathrm{mM}$ imidazole. SDS/PAGE and immunoblot analysis of the purified preparations of the muscle non-polyhistidine-tagged proteins (wild-type, [R136A]M, [E137A]M and [R138A]M mutants) revealed a single $51000-M_{\mathrm{r}}$ band. A single $56600-M_{\mathrm{r}}$ band was found for the liver wild-type- $(\mathrm{H})_{6}$ and for the $[\mathrm{R} 139 \mathrm{~K}] \mathrm{L}-(\mathrm{H})_{6}$ and $[\mathrm{R} 139 \mathrm{Q}] \mathrm{L}-(\mathrm{H})_{6}$ mutants. The $[\mathrm{R} 139 \mathrm{~A}] \mathrm{L}-(\mathrm{H})_{6}$ protein preparation showed the same band with some contaminants and was estimated to be about $50 \%$ pure by scanning the Coomassie Blue-stained gel. Because the [R139A]L-(H) ${ }_{6}$ preparation was not homogeneous, direct structural studies were not conducted on this mutant.

\section{PFK-2 and FBPase-2 activity}

The $\mathrm{pH}$ profiles of PFK-2 activity of the recombinant $\mathrm{M}$ isoenzyme and its mutants were the same and presented an optimal activity at high $\mathrm{pH}$ values $(\mathrm{pH}>8)$, confirming previous work [24]. Figure 2 shows the $\mathrm{pH}$ profile of PFK-2 activity of the recombinant liver wild-type- $(\mathrm{H})_{6}$ and the $[\mathrm{R} 139 \mathrm{~A}] \mathrm{L}-(\mathrm{H})_{6}$, $[\mathrm{R} 139 \mathrm{~K}] \mathrm{L}-(\mathrm{H})_{6}$ and $[\mathrm{R} 139 \mathrm{Q}] \mathrm{L}-(\mathrm{H})_{6}$ mutants. The behaviour of the wild-type $\mathrm{L}$ isoenzyme and the $[\mathrm{R} 139 \mathrm{~K}] \mathrm{L}-(\mathrm{H})_{6}$ mutant was the same and reached an optimal activity at $\mathrm{pH}$ values above 7 . The slight acid-shift of the [R139Q]L-(H) ${ }_{6}$ mutant might correspond to the loss of a positive charge due to the replacement of Arg by Gln. The $\mathrm{pH}$ profile of PFK-2 activity of the [R139A]L$(\mathrm{H})_{6}$ mutant was completely different and presented a maximal activity at $\mathrm{pH}$ values below 6.5 .

The kinetic properties of the wild-type and mutant $\mathrm{M}$ isoenzymes were therefore studied at $\mathrm{pH} 8.5$ as reported $[24,30]$. The changes in kinetic properties induced by mutation of Arg-

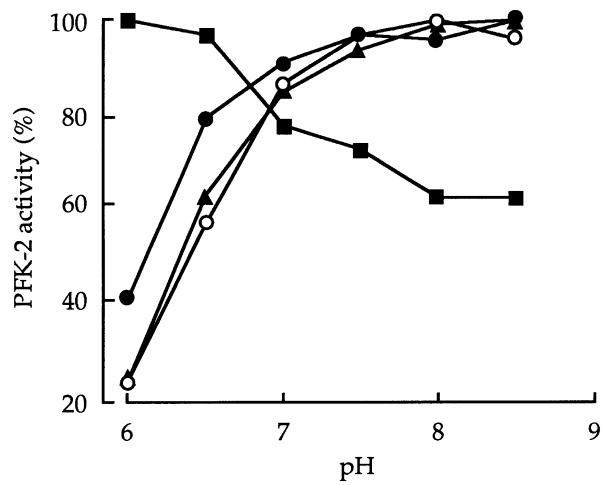

Figure 2 Effect of mutations on the pH profile of PFK-2 activity

PFK-2 activity of the wild-type $\mathrm{L}-(\mathrm{H})_{6}(\mathrm{O})$ isoenzyme was measured at the indicated $\mathrm{pH}$ in the presence of $5 \mathrm{mM} \mathrm{MgATP}$ and $3 \mathrm{mM}$ Fru-6-P. PFK-2 activity of the [R139A]L-(H) 6 isoenzyme was measured in the presence of $20 \mathrm{mM} \mathrm{MgATP}$ and $20 \mathrm{mM}$ Fru-6-P. PFK-2 activities of the $[\mathrm{R} 139 \mathrm{~K}] \mathrm{L}-(\mathrm{H})_{6}(\mathbf{A})$ and $[\mathrm{R} 139 \mathrm{Q}] \mathrm{L}-(\mathrm{H})_{6}(\mathbf{O})$ isoenzymes were measured in the presence of $20 \mathrm{mM}$ MgATP and $5 \mathrm{mM}$ Fru-6-P. These substrate concentrations were chosen to be optimal for each preparation. The values are the means of two determinations. The $100 \%$ values for activity correspond to 70 (wild-type), 0.5 (R139A), 90 (R139K) and 30 (R139Q) munits/mg of protein.

136 and Glu-137 into Ala were quite small in comparison with those produced by mutation of Arg-138 into Ala (Table 2). Mutation of Arg-138 into Ala greatly increased the $K_{\mathrm{m}}$ for Fru6-P (280-fold) and the $\mathrm{IC}_{50}$ for magnesium citrate (15-fold).

We chose to study the kinetic properties of the wild-type and mutant $\mathrm{L}$ isoenzyme at $\mathrm{pH} 7.1$, which was close to the optimal $\mathrm{pH}$ of all the Arg-139 mutants and has been used in previous studies [22]. Mutation of Arg-139 into Ala caused drastic changes in PFK-2 kinetic properties (Table 3). It decreased the $V_{\max }$ (to 60 -fold, taking into account the fact that the preparation was approx. $50 \%$ pure), increased the $K_{\mathrm{m}}$ for Fru-6-P (90-fold) and for $\mathrm{MgATP}$ (10-fold) and increased the $\mathrm{IC}_{50}$ for magnesium citrate (5-fold). Mutation of the same residue into Lys and Gln did not decrease the $V_{\max }$ but also increased the $K_{\mathrm{m}}$ for Fru-6-P (8-fold and 13-fold respectively), for MgATP (20-fold and 25fold respectively), and increased the $\mathrm{IC}_{50}$ for $\mathrm{MgADP}$ (30-fold and 50-fold respectively) and for magnesium citrate (7-fold and 25 -fold respectively). The $V_{\max }$ of $[\mathrm{R} 139 \mathrm{~K}] \mathrm{L}-(\mathrm{H})_{6}$ was approximately doubled.

The mutations caused only minor modifications in the kinetic parameters of FBPase-2 (Tables 2 and 3).

\section{Nucleotide binding}

As reported above, mutation of Arg-139 into Lys or Gln principally affected the $K_{\mathrm{m}}$ for $\mathrm{MgATP}$ and the $\mathrm{IC}_{50}$ for $\mathrm{MgADP}$ of PFK-2. Therefore we measured nucleotide binding by changes in intrinsic fluorescence of the proteins. On addition of nucleotides, quenching of intrinsic fluorescence was measured. On excitation at $295 \mathrm{~nm}$ the intrinsic fluorescence emission spectra of wild-type L-(H) $)_{6}$, $[\mathrm{R} 139 \mathrm{~K}] \mathrm{L}-(\mathrm{H})_{6}$ and $[\mathrm{R} 139 \mathrm{Q}] \mathrm{L}-(\mathrm{H})_{6}$ mutants were similar with a maximum at $325 \mathrm{~nm}$ [22]. The addition of increasing concentrations of nucleotides and nucleotide derivatives quenched the intrinsic fluorescence, which was used to estimate $K_{\mathrm{d}}$ values. The binding constants were unaffected by mutation of Arg-139 into Lys or Gln (Table 4). The $K_{\mathrm{d}}$ values for MgMantATP and MgMantADP were about three orders of magnitude lower than the $K_{\mathrm{m}}$ for MgATP and the $\mathrm{IC}_{50}$ for 


\section{Table 2 Effect of mutations on the kinetic properties of the $M$ isoenzyme}

PFK-2 activity was measured in buffer containing $50 \mathrm{mM}$ Hepes, pH 8.5, $100 \mathrm{mM} \mathrm{KCl,} 20 \mathrm{mM} \mathrm{KF}, 1 \mathrm{mM}$ DTT, $5 \mathrm{mM}$ potassium phosphate and $1 \mathrm{mg} / \mathrm{ml}$ BSA [42]. For the Fru-6-P and MgATP saturation curves, the concentrations of substrates were varied up to 10 times the $K_{\mathrm{m}}$ values, except for the [R138A]M mutant, where the concentration of Fru-6-P could only be varied up to twice the $K_{\mathrm{m}}$. The concentration of the other substrate was 10 times the $K_{\mathrm{m}}$, except for the [R138A]M mutant, where the Fru-6-P concentration was twice the $K_{\mathrm{m}}$. For the $\mathrm{IC}_{50}$ for magnesium citrate, the Fru-6-P and MgATP concentrations were equal to the $K_{\mathrm{m}}$ and 10 times the $K_{\mathrm{m}}$ respectively. FBPase-2 was measured as described [42] at pH 7.1 in the presence of 1 mM potassium phosphate with concentrations of $\left[2-{ }^{32} \mathrm{P}\right] \mathrm{Fru}-2,6-\mathrm{P}_{2}$ up to ten times the $K_{\mathrm{m}}$ value. The $\mathrm{IC}_{50}$ for Fru-6-P was measured in the presence of $5 \mu \mathrm{M}$ Fru-2,6-P. . The values are means $\pm \mathrm{S}$.E.M. for three determinations. *Statistically significant difference $(P<0.01)$ compared with the wild-type.

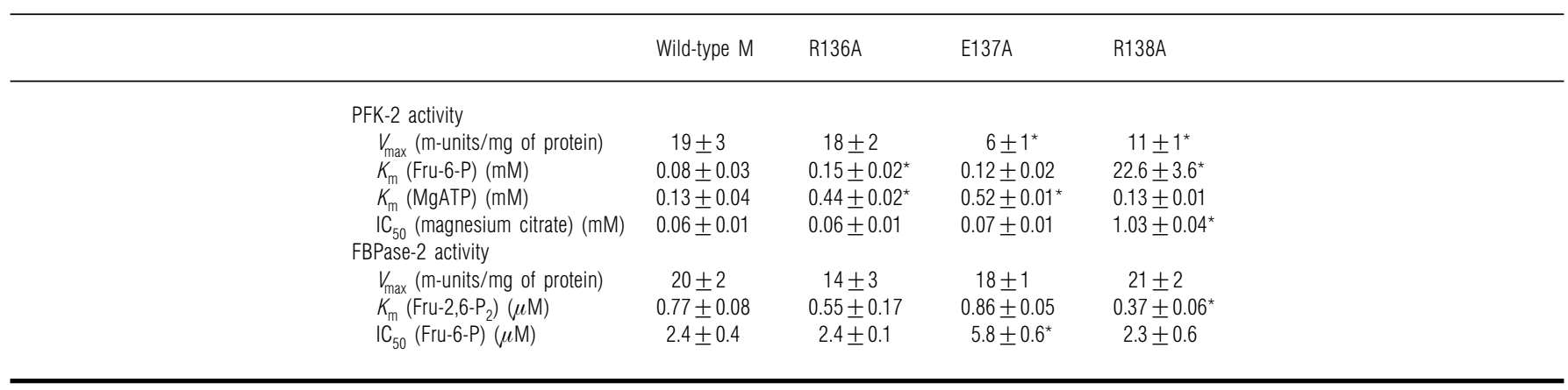

\section{Table 3 Effect of mutations on the kinetic properties of the $L$ isoenzyme}

PFK-2 activity of the polyhistidine-tagged L isoenzyme was measured in buffer containing $50 \mathrm{mM}$ Hepes, $\mathrm{pH}$ 7.1, $100 \mathrm{mM} \mathrm{KCl}, 20 \mathrm{mM} \mathrm{KF}, 1 \mathrm{mM}$ DTT, $5 \mathrm{mM}$ potassium phosphate and $1 \mathrm{mg} / \mathrm{mL}$ $\mathrm{BSA}$ as described in Table 2. FBPase-2 activity was measured as described in Table 2. For the $\mathrm{IC}_{50}$ for $\mathrm{MgADP}$, the MgATP and Fru-6-P concentrations were equal to the $K_{\mathrm{m}}$ and 10 times the $K_{\mathrm{m}}$ respectively. The values are means \pm S.E.M. for three separate determinations. ${ }^{*}$ Statistically significant difference $(P<0.01)$ compared with the wild-type.

Wild-type L R139A R139K R1390

\begin{tabular}{|c|c|c|c|c|}
\hline \multicolumn{5}{|l|}{ PFK-2 activity } \\
\hline$V_{\max }$ (m-units/mg of protein) & $63 \pm 6$ & $0.6 \pm 0.1^{*}$ & $118 \pm 12^{*}$ & $55 \pm 7$ \\
\hline$K_{m}($ Fru-6-P) (mM) & $0.04 \pm 0.01$ & $3.5 \pm 0.4^{*}$ & $0.30 \pm 0.03^{*}$ & $0.53 \pm 0.03^{*}$ \\
\hline$K_{\mathrm{m}}(\mathrm{MgATP})(\mathrm{mM})$ & $0.19 \pm 0.01$ & $2.2 \pm 0.3^{*}$ & $3.7 \pm 0.5^{\star}$ & $5.1 \pm 0.3^{*}$ \\
\hline $\mathrm{IC}_{50}$ (magnesium citrate) (mM) & $0.02 \pm 0.01$ & $0.10 \pm 0.02^{*}$ & $0.14 \pm 0.01^{*}$ & $0.54 \pm 0.05^{\star}$ \\
\hline$I_{50}(\mathrm{MgADP})(\mathrm{mM})$ & $0.07 \pm 0.01$ & $1.91 \pm 0.24^{*}$ & $2.27 \pm 0.38^{*}$ & $3.67 \pm 0.19^{*}$ \\
\hline \multicolumn{5}{|l|}{ FBPase-2 activity } \\
\hline$V_{\max }$ (m-units/mg of protein) & $10 \pm 3$ & $6 \pm 1$ & $9 \pm 1$ & $8 \pm 1$ \\
\hline$K_{m}\left(\right.$ Fru-2,6- $\left.P_{2}\right)(\mu \mathrm{M})$ & $0.48 \pm 0.06$ & $1.68 \pm 0.21^{*}$ & $0.40 \pm 0.08$ & $0.42 \pm 0.08^{*}$ \\
\hline$I_{50}($ Fru-6-P) $(\mu \mathrm{M})$ & $3.0 \pm 1.0$ & $6.4 \pm 1.2^{*}$ & $2.3 \pm 1.1^{*}$ & $2.3 \pm 0.8$ \\
\hline
\end{tabular}

Table 4 Effect of mutations on the binding constants for nucleotides

Fluorescence measurements were performed as indicated in the Materials and methods section. The $K_{\mathrm{d}}$ values correspond to the high-affinity site [22] and were calculated as described in the Materials and methods section. Individual values are shown.

\begin{tabular}{llll}
\hline & Wild-type L & R139K & R1390 \\
\hline$K_{\mathrm{d}}$ (MgATP) (mM) & $0.04,0.05$ & $0.03,0.04$ & $0.04,0.05$ \\
$K_{\mathrm{d}}$ (MgADP) (mM) & $0.06,0.07$ & $0.04,0.05$ & $0.05,0.06$ \\
$K_{\mathrm{d}}$ (MgMantATP) $(\mu \mathrm{M})$ & $0.04,0.05$ & $0.03,0.04$ & $0.03,0.04$ \\
$K_{\mathrm{d}}$ (MgMantADP) $(\mu \mathrm{M})$ & $0.02,0.03$ & $0.01,0.02$ & $0.02,0.03$ \\
\hline
\end{tabular}

MgADP in all cases, in agreement with previous observations on other proteins [22,31-34].

\section{Structures of the wild-type and mutant preparations}

Intrinsic fluorescence was further used to study the structures of the different enzyme preparations indirectly. Reversible subunit dissociation and partial protein unfolding induced by denaturation with $\mathrm{GdmCl}$ were monitored by the quenching of intrinsic fluorescence. The fluorescence emission spectra of the wild-type $\mathrm{L}-(\mathrm{H})_{6}$ and $[\mathrm{R} 139 \mathrm{~K}, \mathrm{Q}] \mathrm{L}-(\mathrm{H})_{6}$ mutants and the wild- type and [R136A]M, [E137A]M and [R138A]M mutant $\mathrm{M}$ isoenzymes were similar, with a maximum at $325 \mathrm{~nm}$ (L isoenzymes) or $335 \mathrm{~nm}$ ( $\mathrm{M}$ isoenzymes). The maximum extent of quenching was $70 \%$ ( $\mathrm{M}$ isoenzymes) and $60 \%$ ( $\mathrm{L}$ isoenzymes). This behaviour reflects the same initial structural environment of tryptophan residues and a similar conformational state of the different mutants compared with the wild-type preparation. Table 5 shows the concentrations of $\mathrm{GdmCl}$ required to produce half-maximal quenching. By this criterion, the mutants were certainly not more susceptible to denaturation by $\mathrm{GdmCl}$ than the wild-types.

Irreversible inactivation of PFK-2 and FBPase-2 by preincubation in $\mathrm{GdmCl}$ was also studied. The $\mathrm{GdmCl}$-induced denaturation and inactivation of PFK-2 differs from the $\mathrm{GdmCl}$ induced quenching of fluorescence as shown by the difference in the dose-response curves for the two phenomena (Figure 3). Table 5 shows the concentrations of $\mathrm{GdmCl}$ corresponding to half-maximal inactivation.

The results (Table 5) indicate that PFK-2 activity of the mutants was not more sensitive to inactivation by $\mathrm{GdmCl}$ than the wild-type. On the contrary, these mutants seemed less prone to inactivation. Similar results were obtained for FBPase-2 activity of the wild-type and the [R139A]L mutants (results not shown).

The secondary structures of the R138A, R139K and R139Q 
Table 5 Effect of mutations on the concentrations of $\mathrm{GdmCl}$ giving halfmaximal quenching of intrinsic fluorescence and PFK-2 inactivation

PFK-2/FBPase-2 preparations were incubated in the presence of $\mathrm{GdmCl}$ as described in the Materials and methods section. PFK-2 activity was assayed at pH 7.1 for the $L$ isoenzymes and 8.5 for the $\mathrm{M}$ isoenzymes under optimal conditions. The concentration of $\mathrm{GdmCl}$ required to obtain $50 \%$ of the maximum effect is represented by $[\mathrm{GdmCl}]_{50}$. The results are the means of two separate determinations. Abbreviation: n.d., not determined.

\begin{tabular}{|c|c|c|}
\hline & \multicolumn{2}{|l|}{$[\mathrm{GdmCl}]_{50}(\mathrm{M})$} \\
\hline & $\begin{array}{l}\text { Quenching of } \\
\text { fluorescence }\end{array}$ & $\begin{array}{l}\text { PFK-2 activity } \\
\text { inactivation }\end{array}$ \\
\hline Wild-type M & 0.28 & 0.52 \\
\hline [R136A]M & 0.57 & 0.70 \\
\hline [E137A]M & 0.44 & 0.69 \\
\hline [R138A]M & 0.48 & 0.68 \\
\hline Wild-type L-(H) 6 & 0.45 & 0.53 \\
\hline$[\mathrm{R} 139 \mathrm{~A}] \mathrm{L}-(\mathrm{H})_{6}$ & n.d. & 1.40 \\
\hline$[\mathrm{R} 139 \mathrm{~K}] \mathrm{L}-(\mathrm{H})_{6}$ & 1.51 & 0.72 \\
\hline$[\mathrm{R} 1390] \mathrm{L}-(\mathrm{H})_{6}$ & 1.61 & 0.70 \\
\hline
\end{tabular}
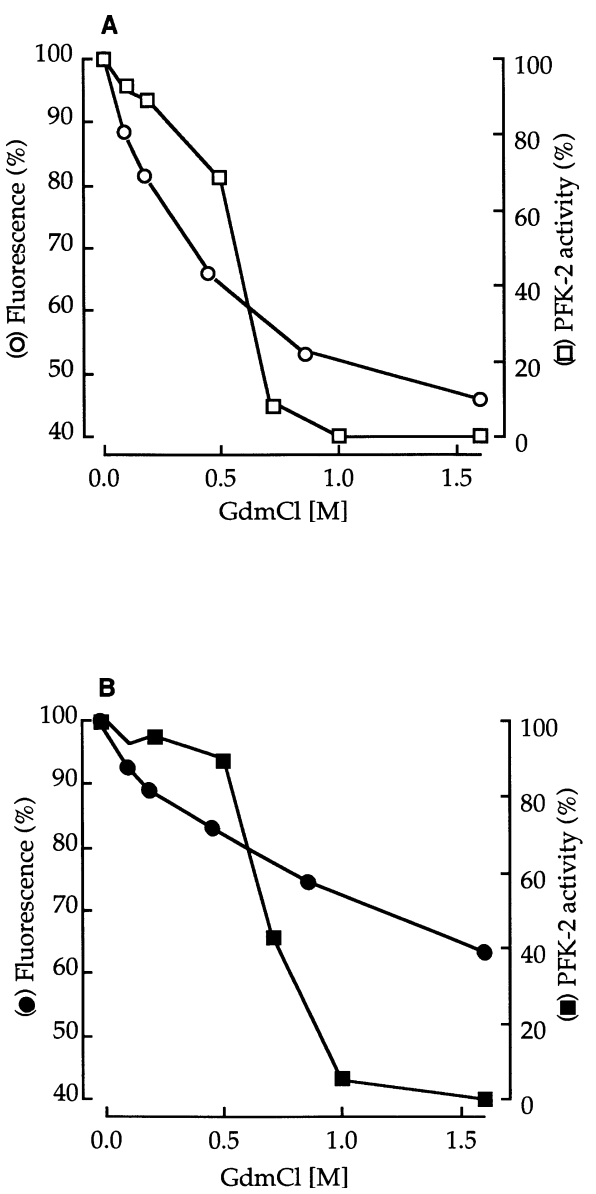

Figure 3 Effect of GdmCl on intrinsic fluorescence and PFK-2 activity of the wild-type L-(H) $)_{6}(A)$ and $[R 1390] L-(H)_{6}(B)$ mutant preparations

The experimental conditions were as described in Table 5. Similar effects were obtained with the other mutations. The values are the means of two determinations. The $100 \%$ values for activity correspond to 70 (wild-type) and 30 (R139Q) m-units/mg of protein.
Table 6 Ratio of the mean residue ellipticity at 222 and $208 \mathrm{~nm}$ measured by CD

Spectra were recorded and $R$ was calculated as described in the Materials and methods section. $R$ is the ratio of the mean residue ellipticities at 222 and $208 \mathrm{~nm}$ and is taken as an index of $\alpha$-helix content [39]. Results are means \pm S.E.M. for the number of determinations shown in parentheses; otherwise individual values are given.

$R$

$\begin{array}{ll}\text { Wild-type M } & 1.01 \pm 0.03(5) \\ {[\text { R138A]M }} & 1.02,1.00 \\ \text { Wild-type L-(H) } & 0.94 \pm 0.03(4) \\ { }_{\text {R139K]L-(H) }} & 0.94,0.98 \\ \text { [R139Q]L-(H) }_{6} & 0.97,0.98\end{array}$

mutants were assessed by CD, which allowed the $\alpha$-helix content of the different preparations to be evaluated. Two minima at 222 and $208 \mathrm{~nm}$ were obtained as described [22] and the ratio $(R)$ of the mean residue ellipticities at 222 and $208 \mathrm{~nm}$ (Table 6) were taken as an index of $\alpha$-helix content [39]. Analysis of the spectra revealed no significant differences between the [R138A]M, $[\mathrm{R} 139 \mathrm{~K}] \mathrm{L}-(\mathrm{H})_{6}$ and $[\mathrm{R} 139 \mathrm{Q}] \mathrm{L}-(\mathrm{H})_{6}$ mutants and the corresponding wild-type enzymes. Therefore the changes in PFK-2 kinetic properties caused by these mutations were not due to a change in $\alpha$-helix content.

\section{DISCUSSION}

Before assigning a role in catalysis or substrate binding to the conserved amino acid residues studied in this work, we verified that the changes in kinetic properties induced by mutagenesis were not due to a change in structure. $\mathrm{CD}$, quenching of intrinsic fluorescence of the protein by $\mathrm{GdmCl}$, and denaturation and inactivation of activity by $\mathrm{GdmCl}$ indicated that there was no mutagenesis-induced change in the structure or instability of PFK-2 in the [R136A]M, [E137A]M, [R138A]M, [R139K]L and [R139Q]L mutants. For the [R139A]L mutant, the study of PFK-2 and FBPase-2 inactivation by $\mathrm{GdmCl}$, together with the fact that the $V_{\max }$ of FBPase-2 was unaffected by the mutation, suggested that the changes in kinetic properties of PFK-2 were probably not due to structural changes.

Arg-136 and Glu-137 are not essential for catalysis or substrate binding, because their mutation into Ala had little effect on the kinetic parameters of PFK-2 and FBPase-2.

The main changes in kinetic parameters resulting from mutation of Arg-138 were in PFK-2 activity. There was a 280 -fold increase in $K_{\mathrm{m}}$ for Fru-6-P and a 15 -fold increase in $\mathrm{IC}_{50}$ for magnesium citrate, with little effect on the $V_{\max }$. These findings indicate that Arg-138 is involved in Fru-6-P binding in the PFK2 domain (Figure 4). This site might also bind magnesium citrate. If so, Arg-138 would play a similar role to that of Arg-104, whose mutation into Ser was also shown to increase the $K_{\mathrm{m}}$ for Fru-6$\mathrm{P}$ and the $\mathrm{IC}_{50}$ for magnesium citrate [21]. Mutation of Arg-138 did not affect the $\mathrm{IC}_{50}$ for Fru-6-P of FBPase-2, confirming the existence of two separate Fru-6-P-binding sites in each catalytic domain. Our results, together with those of other mutations in the PFK-2 domain, indicate that Arg-139, Arg-104, Arg-195 and Arg-225 bind Fru-6-P. Arg-225 might interact through hydrogen bonding [21], whereas the other Arg residues would make electrostatic bonds $[1,21]$. In many proteins, clusters of several positively charged residues are indeed known to interact with negatively charged phosphate. 


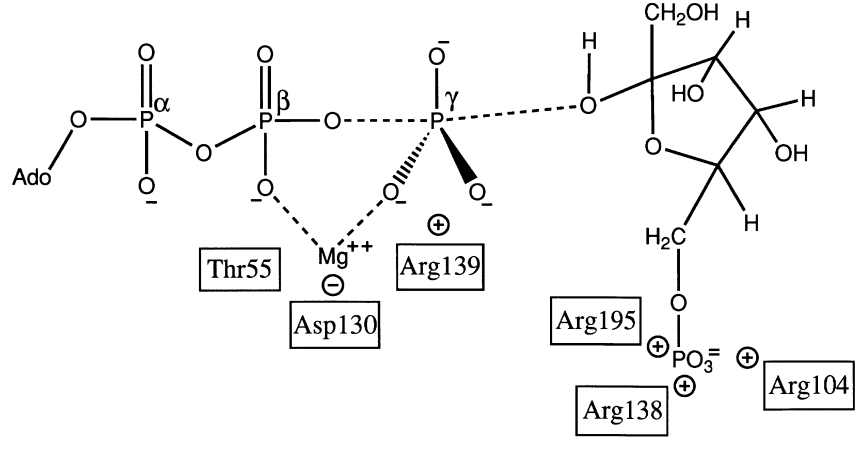

Figure 4 Schematic representation of the transition state of the PFK-2 reaction

Mutation of Arg-139 into Ala mainly affected PFK-2 activity, with little effect on FBPase-2 activity. This mutation not only increased the $K_{\mathrm{m}}$ values for both substrates of PFK-2, but also caused a 60 -fold decrease in the $V_{\max }$, so that the catalytic efficiencies $\left(V_{\max } / K_{\mathrm{m}}\right)$ for MgATP and Fru-6-P were decreased to 600 -fold and 5000 -fold respectively. In addition, the R139A mutation completely changed the $\mathrm{pH}$ profile of PFK-2 activity. Mutation of Arg-139 into Lys increased the $K_{\mathrm{m}}$ and $\mathrm{IC}_{50}$ for adenine nucleotides without changing the $K_{\mathrm{d}}$, and doubled the $V_{\max }$ of PFK-2. Mutation of Arg-139 into Gln had the same effect, except that $V_{\max }$ was unaffected.

Similar modifications of the kinetic properties were induced by mutagenesis of Arg-72 in PFK-1 [40,41] and Asp-130 [24] in PFK-2. They decreased the $V_{\max }$, increased the $K_{\mathrm{m}}$ values and altered the $\mathrm{pH}$ profile. In addition, mutation of Thr-55 to Cys abolished PFK-2 activity, whereas mutation of Thr-55 to Ser doubled the $V_{\max }$ and increased the $K_{\mathrm{m}}$ for MgATP without affecting the $K_{\mathrm{d}}$ for nucleotide binding [22]. These residues have been proposed to be involved in catalysis by stabilizing the transition state. By analogy we propose that Arg-139 interacts directly or indirectly with the $\gamma$-phosphate of ATP in the transition state and thus participates in catalysis (Figure 4). The positively charged Arg residue could compensate for one of the negative charges in the trigonal bipyramid transition state during direct in-line transfer of the $\gamma$-phosphate of ATP to Fru-6-P in the compulsory ordered ternary complex mechanism of PFK-2 [1]. In this mechanism, ATP binds first to the enzyme and ADP release might be rate-limiting. The fact that the mutation of Arg139 to Ala did not completely abolish PFK-2 activity suggests that other residues are involved in stabilization of the transition state, as is known for many enzymes.

We thank Dr. K. C. Crépin for help in the mutagenesis experiments, Dr. G. Deléage of the Institut de Biologie et Chimie des Protéines (CNRS Lyon) for the use of the dichrograph machine and for help with the analysis of the spectra, Dr. G. G. Rousseau for continued interest, and Mr. Y. Marchand for secretarial assistance. We thank Dr. H. Vidal of the Institut National de la Santé et de la Recherche Médicale (INSERM Lyon) for acquisition of the CD spectra. D.V. and L.B. are fellows of the Institute for Scientific Research in Industry and Agriculture, Belgium. M.H.R. is Research Associate of the National Fund for Scientific Research (Belgium). This work was supported by the D.G. Higher Education and Scientific Research - French Community of Belgium, by the Caisse Générale d'Epargne et de Retraite, by the Fund for Medical Scientific Research (Belgium) and by the Accord de Coopération: Communauté Française de Belgique (Belgium) - Institut National de la Santé et de la Recherche Médicale (INSERM France).

\section{REFERENCES}

1 Pilkis, S. J., Claus, T. H., Kurland, I. J. and Lange, A. J. (1995) Annu. Rev. Biochem. 64, 799-835

2 Sakata, J., Abe, Y. and Uyeda, K. (1991) J. Biol. Chem. 266, 15764-15770

3 Watanabe, F., Sakaï, A., Furuya, E. and Uyeda, K. (1994) Biochem. Biophys. Res. Commun. 198, 335-340

4 Kretschmer, M. and Fraenkel, D. G. (1991) Biochemistry 30, 10663-10672

5 Paravicini, G. and Kretschmer, M. (1992) Biochemistry 31, 7126-7133

6 Darville, M. I., Crepin, K. M., Vandekerckhove, J., Van Damme, J., Octave, J. N., Rider, M. H., Marchand, M. J., Hue, L. and Rousseau, G. G. (1987) FEBS Lett. 224 $317-321$

7 Lange, A. J., Raafat El-Maghrabi, M. and Pilkis, S. J. (1991) Arch. Biochem. Biophys. 290, 258-263

8 Algaier, J. and Uyeda, K. (1988) Biochem. Biophys. Res. Commun. 153, 328-333

9 Li, L., Lange, A. J. and Pilkis, S. J. (1993) Biochem. Biophys. Res. Commun. 190, $397-405$

10 Sakai, A., Watanabe, F. and Furuya, E. (1994) Biochem. Biophys. Res. Commun. 198, 1099-1106

11 Crepin, K. M., Darville, M. I., Hue, L. and Rousseau, G. G. (1989) Eur. J. Biochem. 183, 433-440

12 Sakata, J. and Uyeda, K. (1990) Proc. Natl. Acad. Sci. U.S.A. 87, 4951-4955

13 Bazan, J. F., Fletterick, R. J. and Pilkis, S. J. (1989) Proc. Natl. Acad. Sci. U.S.A. 86, 9642-9646

14 Lee, Y. H., Ogata, C., Pflugrath, J. W., Levitt, D. G., Sazma, R., Bangszak, L. J. and Pilkis, S. J. (1996) Biochemistry 35, 6010-6019

15 Pilkis, S. J., Regen, D. M., Stewart, H. B., Pilkis, J., Pate, T. M. and El-Maghrabi, M. R. (1984) J. Biol. Chem. 259, 949-958

16 Tauler, A., Lin, L. and Pilkis, S. J. (1990) J. Biol. Chem. 265, 15617-15622

17 Kitajima, S., Sakakibara, R. and Uyeda, K. (1984) J. Biol. Chem. 259, 6896-6903

18 Kretschmer, M. and Hofmann, E. (1984) Biochem. Biophys. Res. Commun. 124, 793-796

19 Kountz, P. D., Freeman, S., Cook, A. G., El-Maghrabi, M. R., Knowles, J. R. and Pilkis, S. J. (1988) J. Biol. Chem. 263, 16069-16072

20 Li, L., Lin, K., Kurland, I. J., Correia, J. J. and Pilkis, S. J. (1992) J. Biol. Chem. 267, 4386-4393

21 Rider, M. H., Crepin, K. M., De Cloedt, M., Bertrand, L., Vertommen, D. and Hue, L. (1995) Biochem. J. 309, 341-346

22 Vertommen, D., Bertrand, L., Sontag, B., Di Pietro, A., Louckx, M. P., Vidal, H., Hue, L. and Rider, M. H. (1996) J. Biol. Chem. 271, 17875-17880

23 Walker, J. E., Saraste, M., Runswick, M. J. and Gay, N. J. (1982) EMBO J. 8 $945-951$

24 Rider, M. H., Crepin, K. M., De Cloedt, M., Bertrand, L. and Hue, L. (1994) Biochem. J. 300, 111-115

25 Chothia, C. (1976) J. Mol. Biol. 105, 1-14

26 Rose, G. D., Geselowitz, A. R., Lesser, G. L., Lee, R. H. and Zehfus, M. H. (1985) Science 229, 834-838

27 Mead, D. A., Szezesma-Skorupa, E. and Kemper, B. (1986) Prot. Eng. 1, 67-74

28 Sayers, J. R., Schmidt, W. and Eckstein, F. (1988) Nucleic Acids Res. 16, 791-802

29 Sanger, F., Nicklen, S. and Coulson, A. R. (1977) Proc. Natl. Acad. Sci. U.S.A. 74, 5463-5467

30 Crepin, K. M., Vertommen, D., Dom, G., Hue, L. and Rider, M. H. (1993) J. Biol. Chem. 268, 15277-15284

31 Falson, P., Penin, F., Divita, G., Lavergne, J.-P., Di Pietro, A., Goody, R. S. and Gautheron, D. C. (1993) Biochemistry 32, 10387-10397

32 Divita, G., Goody, R. S., Gautheron, D. C. and Di Pietro, A. (1993) J. Biol. Chem. 268, 13178-13186

33 Woodward, S. K. A., Eccleston, J. F. and Greeves, M. A. (1991) Biochemistry 30 $422-430$

34 Moore, K. J. M. and Lohman, T. M. (1994) Biochemistry 33, 14550-14564

35 Bradford, H. (1976) Anal. Biochem. 72, 248-254

36 Bensadoun, A. and Weinstein, D. (1976) Anal. Biochem. 70, 241-250

37 Lowry, O. H., Rosebrough, N. J., Farr, A. L. and Randall, R. J. (1951) J. Biol. Chem. 193, $265-275$

38 Laemmli, U. K. (1970) Nature (London) 227, 680-685

39 Vijayakumar, E. K. S., Sudha, T. S. and Balaram, P. (1984) Biopolymers 23, 877-886

40 Zheng, R. L. and Kemp, R. G. (1994) J. Biol. Chem. 269, 18475-18479

41 Berger, S. A. and Evans, P. R. (1990) Nature (London) 343, 575-576

42 Crepin, K. M., De Cloedt, M., Vertommen, D., Foret, D., Michel, A., Rider, M. H., Rousseau, G. G. and Hue, L. (1992) J. Biol. Chem. 267, 21698-21704 